\title{
A lower bound on the essential dimension of a connected linear group
}

\author{
Philippe Gille and Zinovy Reichstein*
}

\begin{abstract}
Let $G$ be a connected linear algebraic group defined over an algebraically closed field $k$ and $H$ be a finite abelian subgroup of $G$ whose order does not divide char $(k)$. We show that the essential dimension of $G$ is bounded from below by $\operatorname{rank}(H)-\operatorname{rank} C_{G}(H)^{0}$, where $\operatorname{rank} C_{G}(H)^{0}$ denotes the rank of the maximal torus in the centralizer $C_{G}(H)$. This inequality, conjectured by J.-P. Serre, generalizes previous results of Reichstein-Youssin (where char $(k)$ is assumed to be 0 and $C_{G}(H)$ to be finite) and Chernousov-Serre (where $H$ is assumed to be a 2-group).
\end{abstract}

Mathematics Subject Classification (2000). 11E72, 20G10, 14L30.

Keywords. Linear algebraic group, essential dimension, non-abelian cohomology, group action.

\section{Introduction}

Let $k$ be a base field, $K / k$ be a field extension, $G / k$ be a linear algebraic group and $\alpha \in H^{1}(K, G)$ be a $G$-torsor over $\operatorname{Spec}(K)$. We will say that $\alpha$ descends to a subfield $K_{0} \subset K$ if $\alpha$ lies in the image of the natural map $H^{1}\left(K_{0}, G\right) \rightarrow H^{1}(K, G)$. The essential dimension ed $\operatorname{di}_{k}(\alpha)$ of $\alpha$, is defined as the minimal value of $\operatorname{trdeg}_{k}\left(K_{0}\right)$, where $\alpha$ descends to $K_{0}$ and $k \subset K_{0}$. (Throughout this paper we will work over a fixed algebraically closed field $k$; for this reason we will write ed in place of $\mathrm{ed}_{k}$.) We also define $\operatorname{ed}(\alpha ; l)$ as the minimal value of $\operatorname{ed}\left(\alpha_{L}\right)$, as $L / K$ ranges over all finite field extensions of degree prime to $l$. Here $l$ is a prime integer. The essential dimension $\operatorname{ed}(G)$ of the group $G$ (respectively, the essential dimension ed $(G ; l)$ of $G$ at $l$ ) is defined as the maximal value of ed $(\alpha)$ (respectively, of ed $(\alpha ; l)$ ), as $K / k$ ranges over all field extensions and $\alpha$ ranges over $H^{1}(K, G)$. For details on the notion of essential dimension, its various interpretations and numerous examples, see $[\mathrm{Re}],\left[\mathrm{RY}_{1}\right]$ and $[\mathrm{BF}]$.

Many of the best known lower bounds on $\operatorname{ed}(G)$ and $\operatorname{ed}(G ; l)$, for specific groups $G$, are deduced from the following theorem.

\footnotetext{
${ }^{*}$ Z. Reichstein was partially supported by an NSERC research grant.
} 
1.1 Theorem ([RY 1$]$, Theorem 7.8). Let $G$ be a connected semisimple linear algebraic group defined over an algebraically closed field $k$ of characteristic zero and $H$ be a finite abelian subgroup of $G$. Assume that the centralizer $C_{G}(H)$ is finite. Then

(a) $\operatorname{ed}(G) \geq \operatorname{rank}(H)$ and

(b) if $H$ is an $l$-group, then $\operatorname{ed}(G ; l) \geq \operatorname{rank}(H)$.

Here by the rank of a finite abelian group $H$ we mean the smallest positive integer $r$ such that $H$ can be written as a direct product of $r$ cyclic groups. Equivalently, $\operatorname{rank}(H)$ is the minimal dimension of a faithful complex linear representation of $H$.

The purpose of this paper is to prove the following more general inequality conjectured by J.-P. Serre (e-mail, July 25, 2005).

1.2 Theorem. Let $G$ be a connected reductive linear algebraic group defined over an algebraically closed base field $k$. Suppose that $H$ is a finite abelian subgroup of $G$ and char $(k)$ does not divide $|H|$. Then

(a) $\operatorname{ed}(G) \geq \operatorname{rank} H-\operatorname{rank} C_{G}(H)^{0}$.

(b) Moreover, if $H$ is an $l$-group, then $\operatorname{ed}(G ; l) \geq \operatorname{rank} H-\operatorname{rank} C_{G}(H)^{0}$.

Here $C_{G}(H)^{0}$ denotes the connected component of the centralizer of $H$ in $G$, and by the rank of this connected group we mean the dimension of its maximal torus. In particular, if $\operatorname{char}(k)=0$ and the centralizer $C_{G}(H)$ is finite (i.e., rank $C_{G}(H)^{0}=0$ ), then Theorem 1.2 reduces to Theorem 1.1. Note, however, that even in this special case the proof we present here is simpler than the one in $\left[\mathrm{RY}_{1}\right]$; in particular, it does not rely on resolution of singularities.

We also remark that our argument shows a bit more, namely that the essential dimension of a particular torsor, which we call a loop torsor, is $\geq \operatorname{rank}(H)-$ rank $C_{G}(H)^{0}$. Here by a loop torsor we mean the image of a versal $H$-torsor under the natural map $H^{1}(*, H) \rightarrow H^{1}(*, G)$. (Such torsors come up in connection with loop algebras; see [GP].)

Chernousov and Serre [CS] used techniques from the theory of quadratic forms to show that, in the case where $H$ is a 2-group, many of the bounds given by Theorem 1.1 (b) remain valid over any algebraically closed field base field $k$ of characteristic $\neq 2$. The "incompressible" quadratic forms they construct are closely related to loop torsors; our arguments may thus be viewed as extending their approach to abelian subgroups $H$ which are not necessarily 2-groups.

In order to clarify the exposition we will give two proofs of Theorem 1.2. The first one, presented in Section 4, is quite short but it relies on resolution of singularities and, in particular, only works in characteristic zero. The second proof, presented in Section 8 requires a bit more work. The advantage of this more elaborate argument is that it is entirely independent of resolution of singularities; in particular, it works in 
prime characteristic not dividing $|H|$. Both proofs rely, in a key way, on the existence results for wonderful (and regular) group compactifications from [Br] and [BK] (see Section 2) and on the "reduction of structure" Theorem 1.1 from [CGR]. The case where $\operatorname{char}(k)$ divides the order of the Weyl group of $G$ is particularly delicate; here we use a refined version of [CGR, Theorem 1.1], which is proved in [CGR2].

The following symbols will be used for the remainder of the paper.

$k$, algebraically closed base field of characteristic $p \geq 0$;

$G$, connected reductive linear group defined over $k$;

$\bar{G}$, a regular compactification of $G$;

$H$, finite abelian group;

ed, essential dimension over $k$;

$F_{n}=k\left(\left(t_{1}\right)\right) \ldots\left(\left(t_{n}\right)\right)$, iterated Laurent series field in $n$ variables.

\section{Regular compactifications}

Let $G$ be a connected reductive algebraic group defined over $k$. Let $B$ and $B^{-}$ be opposite Borel subgroups of $G$, containing a maximal torus $T$. By the Bruhat decomposition, $G$ has finitely many $B \times B^{-}$orbits. Hence, by [BK, Proposition 6.2.5], $G$ (viewed as a $G \times G$-variety) has a "regular" compactification in the sense of [BDP]; we will denote this compactification by $\bar{G}$. (Note that the terms "regular" and "smooth" are not interchangeable in this context; a regular compactification is smooth but not the other way around.) In particular, if $G$ is adjoint, then $\bar{G}$ is the wonderful compactification of $G$ constructed in [DP] (and in prime characteristic in [St]). Regular compactifications have many interesting special properties; most of them will not be used in the sequel. The only property of $\bar{G}$ we will need is the following description of the stabilizers of points in $\bar{G}$ from [Br, Proposition A.1].

Every $G \times G$ orbit $\mathcal{O}$ in a regular compactification $\bar{G}$ of $G$ has a unique point $\bar{g}$ such that $\left(B \times B^{-}\right) \bar{g}$ is open in $\mathcal{O}$ and $\bar{g}$ is the limit of some 1-parameter subgroup of $T$. We shall refer to $\bar{g}$ as a special point. For example, the special point in the dense orbit $\mathcal{O}=G$ of $\bar{G}$ is the identity element $\bar{g}=e$.

The stabilizer of a special point $\bar{g}$ in $G \times G$ has the following form. Let $P$ be the projection of $\operatorname{Stab}_{G \times G}(\bar{g})$ to the first factor of $G \times G$ and $Q$ be the projection to the second factor. Then $P$ and $Q$ are opposite parabolic subgroups. Denote the unipotent radicals of $P$ and $Q$ by $P_{u}$ and $Q_{u}$ and their common Levi subgroup $P \cap Q$ by $L$. The stabilizer $\operatorname{Stab}_{G \times G}(\bar{g})$ is then given by

$$
\operatorname{Stab}_{G \times G}(\bar{g})=\left\{\left(p_{u} l z, q_{u} l\right) \mid p_{u} \in P_{u}, q_{u} \in Q_{u}, l \in L, z \in Z\right\},
$$

where $Z$ is a subgroup of $Z(L)^{0}$. Of course, the stabilizer of any other point in $\mathcal{O}$ is conjugate to this subgroup. 
2.2 Proposition. Let $\bar{g}$ be a special point in the regular compactification $\bar{G}$ of $G$ and $\Gamma$ be a finite subgroup of $\operatorname{Stab}_{G \times G}(\bar{g})$ whose order is prime to $\operatorname{char}(k)$. Then $\left|\operatorname{rank}\left(\pi_{2}(\Gamma)\right)-\operatorname{rank}\left(\pi_{1}(\Gamma)\right)\right| \leq \operatorname{rank}\left(Z(L)^{0}\right)$.

Here $\pi_{1}, \pi_{2}: G \times G \rightarrow G$ denote the projection to the first and second factor, respectively, $P=\pi_{1} \operatorname{Stab}_{G \times G}(\bar{g}), Q=\pi_{2} \operatorname{Stab}_{G \times G}(\bar{g})$ and $L=P \cap Q$ as above, and $\operatorname{rank}(\Gamma)$ denotes the maximal value of $\operatorname{rank}(A)$, as $A$ ranges over the abelian subgroups of a finite group $\Gamma$.

Proof. The proof is based on tracing $\Gamma$ through the diagram of natural projections

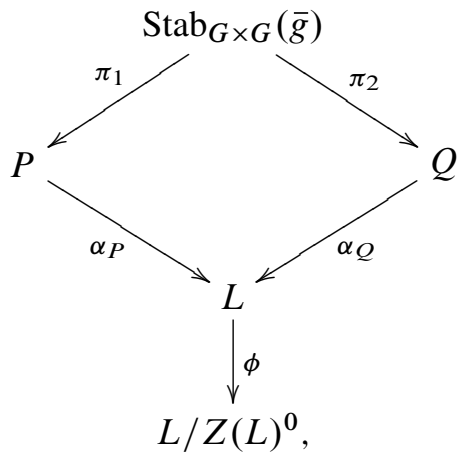

where $\alpha_{P}\left(p_{u} l\right)=l$ and $\alpha_{Q}\left(q_{u} l\right)=l$ for any $p_{u} \in P_{u}, q_{u} \in Q_{u}$ and $l \in L$. Since the kernels $P_{u}$ and $Q_{u}$ of $\alpha_{P}$ and $\alpha_{Q}$ are unipotent, we see that $\alpha_{P}$ and $\alpha_{Q}$ project the finite groups $\pi_{1}(\Gamma)$ and $\pi_{2}(\Gamma)$ isomorphically onto subgroups of $L$, which we will denote by $\Gamma_{1}$ and $\Gamma_{2}$, respectively. By (2.1), $\Gamma_{1}$ and $\Gamma_{2}$ have the same image in $L / Z(L)^{0}$, which we will denote by $\Gamma_{0}$. Since the natural projection $\phi_{\mid \Gamma_{2}}: \Gamma_{2} \rightarrow \Gamma_{0}$ is surjective with kernel $Z_{2} \subset Z(L)^{0}$, we have

$$
\operatorname{rank}\left(\Gamma_{2}\right) \leq \operatorname{rank}\left(\Gamma_{1}\right)+\operatorname{rank}\left(Z_{2}\right) \leq \operatorname{rank}\left(\Gamma_{1}\right)+\operatorname{rank} Z(L)^{0} .
$$

By symmetry, we also have the reverse inequality $\operatorname{rank}\left(\Gamma_{1}\right)-\operatorname{rank}\left(\Gamma_{2}\right) \leq \operatorname{rank} Z(L)^{0}$, and the proposition follows.

\section{Compactifications of homogeneous spaces}

The following lemma is well known in characteristic zero (see [RY, Lemma 2.1]).

3.1 Lemma. Let $\Gamma$ be a finite group and let $X$ be a normal quasiprojective $\Gamma$-variety. Then

(a) $X$ is covered by affine open $\Gamma$-invariants subsets. 
Assume moreover that the order of $\Gamma$ is invertible in $k$. Then

(b) there is a geometric quotient map $\pi: X \rightarrow X / \Gamma$.

(c) Moreover, if $X$ is projective, then so is $X / \Gamma$.

Recall that we are assuming throughout that the base field $k$ is algebraically closed.

Proof. (a) The proof of loc.cit. is characteristic free.

(b) Recall first that the group $\Gamma$ is linearly reductive, see [MFK, §1]. If $X$ is affine, part (b) is proved in [MFK, Theorem 1.1 and Amplification 1.3]. The general case follows from part (a) and the characteristic free glueing assertion in [PV, Theorem 4.14].

(c) See [N, Theorem 3.14].

Let $G / k$ be a connected reductive linear algebraic group, $\bar{G}$ be a regular compactification, and $\Gamma$ be a finite subgroup of $G$ of order prime to char $(k)$. We shall denote by $\bar{G} / \Gamma$ the geometric quotient of $\bar{G}$ for the action of the finite group $\Gamma$ (on the right). It may be viewed as a (possibly singular) compactification of the homogeneous space $G / \Gamma$. By the properties of geometric quotients

(i) the fibers of the natural projection $\bar{G} \rightarrow \bar{G} / \Gamma$ are the $\Gamma$-orbits of the right action of $\Gamma$ on $\bar{G}$, and

(ii) the left action of $G$ on $\bar{G}$ descends to $\bar{G} / \Gamma$.

3.2 Proposition. Let $G$ be a connected reductive group, $\bar{G}$ be a regular compactification and $\Gamma_{1}, \Gamma_{2}$ be finite subgroups of $G$ whose orders are not divisible by char $(k)$. If $\bar{G} / \Gamma_{2}$ has an $\Gamma_{1}$-fixed point, then

$$
\operatorname{rank}\left(\Gamma_{1}\right)-\operatorname{rank}\left(\Gamma_{2}\right) \leq \operatorname{rank} C_{G}\left(\Gamma_{1}\right)^{0} .
$$

Proof. Let $y$ be an $\Gamma_{1}$-fixed point of $\bar{G} / \Gamma_{2}$ and $x$ be a point of $\bar{G}$ lying above $y$. After translating $x$ by a suitable element $\left(h_{1}, h_{2}\right) \in G \times G$ and replacing $\Gamma_{1}, \Gamma_{2}$ by $h_{1} \Gamma_{1} h_{1}^{-1}, h_{2} \Gamma_{2} h_{2}^{-1}$ respectively, we may assume that $x$ is a special point. Then $P=\pi_{1}\left(\operatorname{Stab}_{G \times G}(x)\right), Q=\pi_{2}\left(\operatorname{Stab}_{G \times G}(x)\right)$ is a pair of opposite parabolics, as in the previous section. Letting $L=P \cap Q$ be their common Levi subgroup, we see that $\operatorname{Stab}_{G \times G}(x)$ is as in (2.1). (Here, as before, $\pi_{i}: G \times G \rightarrow G$ denotes the projection to the $i$ th factor, where $i=1$ or 2.)

Let $\Gamma=\operatorname{Stab}_{\Gamma_{1} \times \Gamma_{2}}(x)$. The fact that $y$ is $\Gamma_{1}$-fixed means that for every $g_{1} \in \Gamma_{1}$, there is an $g_{2} \in \Gamma_{2}$ such that $\left(g_{1}, g_{2}\right) \in \operatorname{Stab}_{G \times G}(x)$. In other words, $\pi_{1}(\Gamma)=\Gamma_{1}$ and, in particular, $\Gamma_{1} \subset P$. By Proposition 2.2

$$
\operatorname{rank}\left(\Gamma_{1}\right)-\operatorname{rank}\left(\pi_{2}(\Gamma)\right) \leq \operatorname{rank} Z(L)^{0} .
$$


Since $\operatorname{rank}\left(\Gamma_{2}\right) \geq \operatorname{rank}\left(\pi_{2}(\Gamma)\right)$, we have

$$
\operatorname{rank}\left(\Gamma_{1}\right)-\operatorname{rank}\left(\Gamma_{2}\right) \leq \operatorname{rank} Z(L)^{0} .
$$

It remains to show that

$$
\operatorname{rank} Z(L)^{0} \leq \operatorname{rank} C_{G}\left(\Gamma_{1}\right)^{0} .
$$

By the Levi decomposition, $P$ is isomorphic to a semidirect product $P_{u} \rtimes L$; see, e.g., $[\mathrm{H}, 30.2]$. Now recall that we are assuming that $\operatorname{char}(k)$ does not divide the order of $\Gamma_{1}$. In particular, $\Gamma_{1}$ is linearly reductive. Thus by [J, Lemma 11.24], $\Gamma_{1}$ is conjugate to a finite subgroup of $L$. Denote this subgroup by $\Gamma_{L}$. The connected centralizers $C_{G}\left(\Gamma_{1}\right)^{0}$ and $C_{G}\left(\Gamma_{L}\right)^{0}$ will then also be conjugate in $G$, and since $Z(L)^{0} \subset C_{G}\left(\Gamma_{L}\right)^{0}$, we see that

$$
\operatorname{rank} C_{G}\left(\Gamma_{1}\right)^{0}=\operatorname{rank} C_{G}\left(\Gamma_{L}\right)^{0} \geq \operatorname{rank} Z(L)^{0},
$$

as claimed.

\section{Proof of Theorem 1.2 in characteristic zero}

The following lemma is well known; we supply a short proof for lack of a direct reference.

4.1 Lemma. Consider a faithful action of a finite abelian group A on an irreducible algebraic variety $X$, defined over a field $k$. Assume $\operatorname{char}(k)$ does not divide $|A|$. If $A$ fixes a smooth $k$-point in $X$, then $\operatorname{dim}(X) \geq \operatorname{rank}(A)$.

Proof. Let $x \in X(k)$ be a smooth $A$-fixed $k$-point. Then $A$ acts on the regular local ring $R=\mathcal{O}_{x}(X)$ and on its maximal ideal $M=\mathcal{M}_{x}(X)$.

Assume the contrary; $\operatorname{dim}(X)=d$ and $\operatorname{rank}(A)>d$. Then the $A$-representation on the $d$-dimensional cotangent space $T_{x}(X)^{*}=M / M^{2}$ cannot be faithful; denote its kernel by $A_{0} \neq\{1\}$. Since $|A|$ is prime to $\operatorname{char}(k)$, the map $M \rightarrow M / M^{2}$ of $A$-representations splits. Thus $R$ has a system of local parameters $t_{1}, \ldots, t_{d} \in M$ such that each $t_{i}$ is fixed by $A_{0}$. Then $A_{0}$ acts trivially on the completion $\hat{R}=$ $k\left[\left[t_{1}, \ldots, t_{d}\right]\right]$, hence, on $R \subset \hat{R}$. Since $X$ is irreducible, $A_{0}$ acts trivially on $X$. This contradicts our assumption that the $A$-action on $X$ is faithful.

For the remainder of this section we will assume that $k$ is an algebraically closed field of characteristic zero. Before proceeding with the proof of Theorem 1.2, we recall that every $\Gamma$-variety is birationally isomorphic to a smooth projective $\Gamma$-variety; cf. [ $\mathrm{RY}_{2}$, Proposition 2.2]. Here $\Gamma$ is an arbitrary linear algebraic group, not necessarily connected. This fact, whose proof relies on equivariant resolution of singularities 
and thus requires the assumption that $\operatorname{char}(k)=0$, will be used repeatedly for the remainder of this section.

Let $\Gamma$ be a linear algebraic group defined over $k$ and $K / k$ be a finitely generated field extension. Recall that elements of $H^{1}(K, \Gamma)$ are in a natural 1-1 correspondence with birational isomorphism classes of generically free primitive $\Gamma$-varieties $Z$, with $K=k(Z)^{\Gamma}$; see, e.g., [Po, Section 1.3]. Here by saying that $Z$ is a primitive $\Gamma$ variety, we mean that $\Gamma$ transitively permutes the irreducible components of $Y$. We will denote the class of the generically free $\Gamma$-variety $Z$ in $H^{1}(K, \Gamma)$ by $[Z]$.

4.2 Lemma. Every primitive generically free $G$-variety $X$ is birationally isomorphic to a projective $G$-variety of the form $(\bar{G} \times Y) / S$, where

(a) $S$ is a finite subgroup of $G$,

(b) $Y$ is a smooth irreducible projective $S$-variety,

(c) $S$ acts on $\bar{G} \times Y$ by $s \cdot(h, y)=\left(h s^{-1}, s \cdot y\right)$, and $(\bar{G} \times Y) / S$ is the geometric quotient for this action.

Note that in (c) $S$ is a finite group acting on the smooth projective variety $\bar{G} \times Y$. In this situation a geometric quotient exists and is projective; see, e.g., [PV, Theorem 4.14] or Lemma 3.1. Moreover, the natural (left) $G$-action on $\bar{G} \times Y$ (via the first factor) commutes with the $S$-action and thus descends to $(\bar{G} \times Y) / S$. In the statement of the lemma we view $(\bar{G} \times Y) / S$ as a $G$-variety with respect to this action.

Proof. Let $K=k(X)^{G}$. By [CGR, Theorem 1.1] there exists a finite subgroup $S \subset G$ and a generically free primitive $S$-variety $Y$ such that $[X]$ is the image of $[Y] \in H^{1}(K, S)$ under the natural map $H^{1}(K, S) \rightarrow H^{1}(K, G)$. In other words, $X$ is birationally isomorphic to $(G \times Y) / S$. Since the $S$-variety $Y$ is only defined up to birational isomorphism, we may assume without loss of generality that it is smooth and projective. Moreover, $(G \times Y) / S$ is birationally isomorphic to $(\bar{G} \times Y) / S$, as in (c).

In general, the above construction produces only a primitive $S$-variety $Y$. However, it can be slightly modified to ensure that $Y$ is irreducible, as follows. Let $Y_{0}$ be an irreducible component of $Y$ and $S_{0}$ be the subgroup of $S$ consisting of elements that leave $Y_{0}$ invariant. Then it is easy to see that $[Y]$ is the image of $\left[Y_{0}\right]$ under the natural map $H^{1}\left(K, S_{0}\right) \rightarrow H^{1}(K, S)$; cf. e.g., [Re, Example 2.10]. Thus after replacing $S$ by $S_{0}$ and $Y$ by $Y_{0}$, we may assume that $Y$ is irreducible.

4.3 Lemma. Let $G$ be a reductive group, $H \subset G$ be a finite abelian subgroup, and $X$ be a primitive generically free $G$-variety. If $H$ fixes a smooth $k$-point of $X$, then

$$
\operatorname{dim} k(X)^{G} \geq \operatorname{rank}(H)-\operatorname{rank} C_{G}(H)^{0} .
$$


Proof. By Lemma 4.2 there exists a birational $G$-equivariant isomorphism $X \stackrel{\simeq}{\underset{-\rightarrow}{\longrightarrow}}$ $(\bar{G} \times Y) / S$, where $Y$ is a smooth complete $S$-variety and $S \subset G$ is a finite subgroup, as in Lemma 4.2. Note that $\operatorname{dim}(Y)=\operatorname{trdeg}_{k} k(X)^{G}$; we will denote this number by $d$. By the Going Down Theorem [RY ${ }_{1}$, Proposition A.2] $(\bar{G} \times Y) / S$ also has an $H$-fixed $k$-point; denote it by $x=[\bar{g}, y]$. The fiber of the natural projection $X=(\bar{G} \times Y) / S \rightarrow Y / S$ containing $x$ is easily seen to be $G$-equivariantly isomorphic to $\bar{G} / S_{0}$, where $S_{0}:=\operatorname{Stab}_{S}(y)$. Now observe that since $y$ is a smooth $k$-point of $Y$, $S_{0}$ can contain no abelian subgroup of rank $\geq d$; see Lemma 4.1. In other words, $\operatorname{rank}\left(S_{0}\right) \leq d$. By Proposition 3.2,

$$
\operatorname{rank}(H)-\operatorname{rank}\left(S_{0}\right) \leq \operatorname{rank} C_{G}(H)^{0}
$$

and thus

$$
d \geq \operatorname{rank}\left(S_{0}\right) \geq \operatorname{rank}(H)-\operatorname{rank} C_{G}(H)^{0} .
$$

This completes the proof of Lemma 4.3.

We are now ready to proceed with the proof of Theorem 1.2 (in characteristic zero). Let $V$ be a generically free linear $k$-representation of $G$.

(a) The essential dimension $\operatorname{ed}(G)$ is the minimal value of $\operatorname{trdeg}_{k} k(X)^{G}$, where the minimum is taken over all dominant rational $G$-equivariant maps

$$
V \rightarrow X
$$

such that $X$ is a generically free $G$-variety; see [Re, Section 3]. Thus our goal is to show that

$$
\operatorname{trdeg}_{k} k(X)^{G} \geq \operatorname{rank}(H)-\operatorname{rank} C_{G}(H)^{0} .
$$

After replacing $X$ by a birationally equivalent $G$-variety, we may assume that $X$ is smooth and projective. Since $V$ has a smooth $H$-fixed $k$-point (namely the origin), the Going Down Theorem [RY ${ }_{1}$, Proposition A.2] tells us that $X$ also has an $H$-fixed $k$-point (which is smooth, because every $k$-point of $X$ is smooth). The inequality (4.4) now follows from Lemma 4.3.

(b) It suffices to show that the inequality (4.4) holds if there is a diagram of dominant rational $G$-equivariant maps of the form

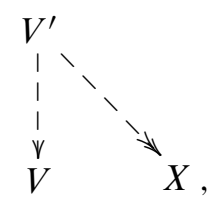

where $X$ is a generically free $G$-variety, $\operatorname{dim}\left(V^{\prime}\right)=\operatorname{dim}(V)$ and $\left[k\left(V^{\prime}\right): k(V)\right]$ is prime to $l$. (Note that the $G$-variety $V^{\prime}$ is not required to be linear.) Once again, we 
may assume without loss of generality that $V^{\prime}$ and $X$ are smooth and complete. Since $H$ fixes the origin in $V$, the Going Up Theorem [RY, Proposition A.4] tells us that $V^{\prime}$ has an $H$-fixed $k$-point. Now by the Going Down Theorem [RY ${ }_{1}$, Proposition A.2], $X$ has an $H$-fixed $k$-point as well, and Lemma 4.3 completes the proof.

\section{The field of iterated Laurent series}

In this section we will describe the structure of the iterated Laurent polynomial field $F_{n}=k\left(\left(t_{1}\right)\right) \ldots\left(\left(t_{n}\right)\right)$. Our proof of Theorem 1.2 in full generality (i.e., without assuming that $\operatorname{char}(k)=0$ ) will make use of these results.

We begin by describing the absolute Galois group $\operatorname{Gal}\left(F_{n}\right)$. Since $F_{n}=F_{n-1}\left(\left(t_{n}\right)\right)$ is a complete valuated field with residue field $F_{n-1}$, we have an exact sequence

$$
1 \rightarrow I_{n} \rightarrow \operatorname{Gal}\left(F_{n}\right) \stackrel{\pi_{n}}{\longrightarrow} \operatorname{Gal}\left(F_{n-1}\right) \rightarrow 1
$$

where $I_{n}$ stands for the inertia group; see [GMS, §II.7]. Let $\widehat{\mathbb{Z}}^{\prime}$ be the prime to $p$ part of $\widehat{\mathbb{Z}}$, i.e. $\widehat{\mathbb{Z}}^{\prime}=\prod_{q \neq p} \widehat{\mathbb{Z}}_{q}$. In particular, if $p=0$, then $\widehat{\mathbb{Z}}^{\prime}=\widehat{\mathbb{Z}}$.

5.1 Lemma. There is a split exact sequence

$$
1 \rightarrow J_{n} \rightarrow \operatorname{Gal}\left(F_{n}\right) \rightarrow\left(\widehat{\mathbb{Z}}^{\prime}\right)^{n} \rightarrow 1,
$$

such that

(1) $J_{n}=1$ if $p=0$,

(2) $J_{n}$ is a free pro- $p$-group if $p>0$.

Proof. We proceed by induction on $n$. The group $I_{n}$ fits in an exact sequence

$$
0 \rightarrow I_{n}^{\text {wild }} \rightarrow I_{n} \rightarrow \hat{\mathbb{Z}}^{\prime} \rightarrow 1,
$$

where $I_{n}^{\text {wild }}$ is the wild inertia group (it is a pro- $p$-group). Define

$$
F_{n, m}:=k\left(\left(\sqrt[m]{t_{1}}\right)\right) \ldots\left(\left(\sqrt[m]{t_{n}}\right)\right) \quad \text { and } \quad F_{n, \infty}:=\underset{(m, p)=1}{\underset{\lim }{\longrightarrow}} F_{n, m} .
$$

Since

$$
\operatorname{Gal}\left(F_{n, \infty} / F_{n-1, \infty}\left(\left(t_{n}\right)\right)\right)=\widehat{\mathbb{Z}}^{\prime},
$$


we have the following commutative diagram of profinite groups:

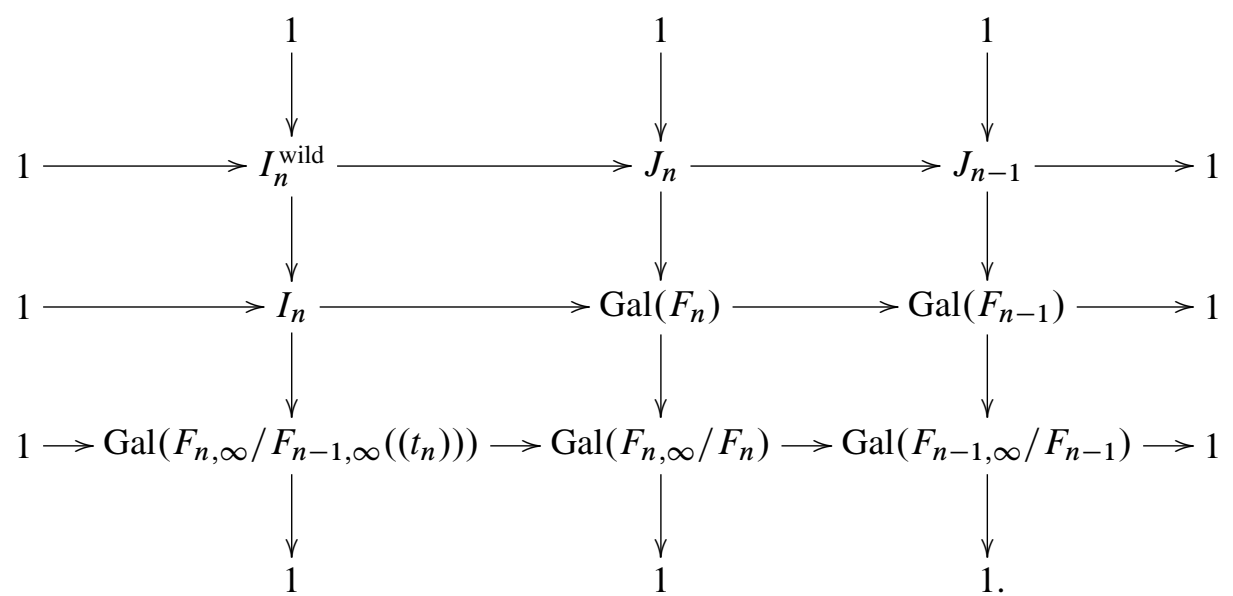

If $p=0$, then $I_{n}^{\text {wild }}=1$; cf. [GMS, §II.7.1]. Hence, the top row in the above diagram tells us that $J_{0} \cong J_{1} \cong J_{2} \cong \ldots$ On the other hand, since $F_{0}=F_{0, \infty}=k$, we see that $J_{0}=\{1\}$. We thus conclude that $J_{n}=1$ for every $n$. (This also follows from [GP, Corollary 2.10].)

Similarly if $p>0$, then $I_{n}^{\text {wild }}$ is a pro- $p$ group (once again, cf. [GMS, §II.7.1]), and we see by induction that $J_{n}$ is pro- $p$-group. The group $J_{n}$ is the absolute Galois group of the field $F_{n, \infty}, \operatorname{socd}_{p}\left(J_{n}\right) \leq 1$ ([Se, II.3.1, Proposition 7]) and $J_{n}$ is then a free pro- $p$-group (ibid, I.4.2, Corollary 2 ).

Finally, since $\left(\widehat{\mathbb{Z}}^{\prime}\right)^{n}$ is a prime-to- $p$ group, we conclude that the sequence (5.2) splits; see [Se, I.5.9, Corollary 1].

We will now show that every finite field extension of $F_{n}$ is $k$-isomorphic to $F_{n}$. Recall that the lexicographic order $\prec$ on $\mathbb{Z}^{n}$ is defined as follows:

$$
\left(m_{1}, \ldots m_{n}\right) \prec\left(m_{1}^{\prime}, \ldots m_{n}^{\prime}\right)
$$

if $m_{i}<m_{i}^{\prime}$ for the smallest subscript $i$ with $m_{i} \neq m_{i}^{\prime}$. A valuation

$$
v=\left(v^{(n)}, \ldots, v^{(1)}\right): E^{\times} \rightarrow \Gamma
$$

on a field $E$ is called $n$-discrete if the group $\Gamma$ is isomorphic to a (lexicographically ordered) subgroup of $\mathbb{Z}^{n}$; see [F, 1.1.3]. Then $E$ is a 1-discrete valuation with respect to the first component $v^{(n)}$ of $v$ and the residue field $E_{n-1}$ is a $(n-1)$-discrete valuation via $\left(v^{(n-1)}, \ldots, v^{(1)}\right)$. In this way we obtain a sequence of fields $E=E_{n}, E_{n-1}, \ldots$, $E_{1}$ such that $E_{i}$ is the residue field of $E_{i+1}$ with respect to a 1-discrete valuation. The residue field $E_{0}$ of $E_{1}$ then coincides with the residue field $\bar{E}_{v}$. 
The definition of completeness for $E$ is inductive as follows [F, 1.2.1]. The field $E$ is a complete $n$-discrete field if $E_{n}$ is complete with respect to $v^{(n)}$ and $E_{n-1}$ is complete. Assume from now on that $E$ is a complete $n$-discrete field. Then according to [W, 3.1], $E$ is henselian, i.e. its valuation ring is a henselian ring. In particular, given a finite extension $E^{\prime} / E$ the valuation $v$ extends uniquely to $v^{\prime}: E^{\prime \times} \rightarrow \frac{1}{\left[E^{\prime}: E\right]} \mathbb{Z}^{n}$, the formula being $v^{\prime}=\frac{1}{\left[E^{\prime}: E\right]} v \circ N_{E^{\prime} / E}$. Then $E^{\prime}$ is a $n$-discrete field which is complete (by induction).

The field $F_{n}=k\left(\left(t_{1}\right)\right)\left(\left(t_{2}\right)\right) \ldots\left(\left(t_{n}\right)\right)$ over iterated Laurent series over a base field $k$ is $n$-complete; see [W, §3]. Here the valuation $v$ on $F_{n}$ is defined by

$$
v\left(\sum_{i_{1}} \ldots \sum_{i_{n}} c_{i_{1}, \ldots, i_{n}} t_{1}^{i_{1}} \ldots t_{n}^{i_{n}}\right)=\operatorname{Min}\left\{\left(i_{1}, \ldots, i_{n}\right) \mid c_{i_{1}, \ldots, i_{n}} \neq 0\right\} .
$$

5.3 Proposition. Let $E$ be a complete $n$-discrete $k$-field. Then

(1) E is isomorphic to $\bar{E}_{v}\left(\left(t_{1}\right)\right)\left(\left(t_{2}\right)\right) \ldots\left(\left(t_{n}\right)\right)$;

(2) Suppose the valuation $v$ is trivial on a perfect subfield $K$ of $E$. Then $E$ is $K$-isomorphic to $\bar{E}_{v}\left(\left(t_{1}\right)\right)\left(\left(t_{2}\right)\right) \ldots\left(\left(t_{n}\right)\right)$.

Proof. (1) immediately follows from (2) if we take $K$ to be the prime subfield of $E$.

To prove (2), first assume that $n=1$. In this case Cohen's structure theorem [Co, Theorem 10] shows that the valuation ring of $E$ is $K$-isomorphic to $\bar{E}_{v}\left[\left[t_{1}\right]\right]$. Thus $E$ is $K$-isomorphic to $\bar{E}_{v}\left(\left(t_{1}\right)\right)$.

Now suppose that $n \geq 2$. Let $E=E_{n}, E_{n-1}, \ldots, E_{1}$ be the sequence of fields constructed above. By induction, we may assume that $E_{n-1}$ is $K$-isomorphic to $\bar{E}_{v}\left(\left(t_{1}\right)\right)\left(\left(t_{2}\right)\right) \ldots\left(\left(t_{n-1}\right)\right)$. Since we know part (2) holds for $n=1$, we conclude that $E=E_{n}$ is $K$-isomorphic to $\bar{E}_{v}\left(\left(t_{1}\right)\right)\left(\left(t_{2}\right)\right) \ldots\left(\left(t_{n}\right)\right)$.

5.4 Corollary. Assume that $k$ is algebraically closed. Then any finite extension of $F_{n}=k\left(\left(t_{1}\right)\right) \ldots\left(\left(t_{n}\right)\right)$ is $k$-isomorphic to $F_{n}$.

Proof. A finite extension $E$ of $F_{n}$ is a complete $n$-discrete field. Its residue field $E_{v}$ is a finite extension of $k$. Since $k$ is algebraically closed, we conclude that $E_{v}=k$. Proposition 5.3 (2) now shows that $E$ is $k$-isomorphic to $F_{n}$.

\section{Reduction of structure}

In this section $G / k$ will denote a linear algebraic group defined over an algebraically closed field $k$ of characteristic $\geq 0$, whose identity component $G^{0}$ is reductive. $F_{n}$ will denote the iterated power series field $k\left(\left(t_{1}\right)\right)\left(\left(t_{2}\right)\right) \ldots\left(\left(t_{n}\right)\right)$ in variables $t_{1}, \ldots, t_{n}$, as in the previous section. As usual, we will say that $\gamma \in H^{1}(K, G)$ 
- descends to a subfield $K_{0} \subset K$ if $\gamma$ lies in the image of the restriction map $H^{1}\left(K_{0}, G\right) \rightarrow H^{1}(K, G)$,

- admits reduction of structure to a subgroup $A \subset G$ if $\gamma$ lies in the image of the natural map $H^{1}(K, A) \rightarrow H^{1}(K, G)$.

6.1 Proposition. Suppose $\gamma \in H^{1}\left(F_{n}, G\right)$ descends to a subfield $K \subset F_{n}$ such that $\operatorname{trdeg}_{k}(K)=d<\infty$.

(a) Assume that $\operatorname{char}(k)=0$. Then $\gamma$ admits reduction of structure to a finite abelian subgroup $A \subset G$ of rank $\leq d$.

(b) Assume that char $(k)=p>0$. Then there exists a finite field extension $F^{\prime} / F_{n}$ such that $\left[F^{\prime}: F_{n}\right]$ is a power of $p$ and $\gamma_{F^{\prime}}$ admits reduction of structure to a finite abelian subgroup $A \subset G$ of rank $\leq d$, whose order $|A|$ is prime to $p$.

Our proof of Proposition 6.1 will make use of the following two simple lemmas.

6.2 Lemma. Suppose $K \subset E$ is a field extension such that $K$ is algebraically closed in E. Then

(a) for every finite Galois field extension $K^{\prime} / K, K_{E}^{\prime}=K^{\prime} \otimes_{K} E$ is a field.

(b) The absolute Galois group $\operatorname{Gal}(K)$ is a quotient of $\operatorname{Gal}(E)$.

Proof. (a) By the primitive element theorem we can write $K^{\prime}$ as $K[x] /(p(x))$ for some irreducible monic polynomial $p(x) \in K[x]$. Then $K_{E}^{\prime}=K^{\prime} \otimes_{K} E=E[x] /(p(x))$, and we need to show that $p(x)$ remains irreducible over $E$.

We argue by contradiction. Suppose $p(x)=p_{1}(x) p_{2}(x)$ for some non-constant monic polynomials $p_{1}(x), p_{2}(x) \in E[x]$. The coefficients of $p_{i}(x)$ are then polynomials in the roots of $p(x)$. (Here $i=1$ or 2.) In particular, they are algebraic over $K$. Since $K$ is algebraically closed in $E$, we conclude that $p_{i}(x) \in K[x]$. Thus $p(x)$ is reducible over $K$, a contradiction.

(b) Let $\bar{K}$ be the algebraic closure of $K$. Then $\operatorname{Gal}(K)=\operatorname{Gal}(\bar{K} / K)=$ $\operatorname{Gal}\left(\bar{K}_{E} / E\right)$, and $\bar{K}_{E}=\bar{K} \otimes_{K} E$ is an $E$-subfield of $\bar{E}$ by part (a).

6.3 Lemma. Let $d \geq 0$ be an integer. Let $\Gamma$ be a finitely generated abelian profinite group such that $\operatorname{cd}(\bar{\Gamma}) \leq d$. Then $\Gamma$ is a direct summand of $\widehat{\mathbb{Z}}^{d}$.

Proof. Without loss of generality, we may assume that $\Gamma$ is a $p$-profinite group for a prime $p$. Since $\operatorname{cd}(\Gamma)<\infty$, we see that $\Gamma$ is torsion free, and since $\Gamma$ is finitely generated and abelian, we conclude that $\Gamma \cong \mathbb{Z}_{p}^{m}$ for some integer $m$; see [RZ, Theorem 4.3.4. (a)]. Thus $\operatorname{cd}(\Gamma)=m$. Since we are assuming $m \leq d$, this shows that $\Gamma$ is a direct summand of $\widehat{\mathbb{Z}}^{d}$. 
Proof of Proposition 6.1. Let $W$ be the Weyl group of $G$. We recall that there exists a finite subgroup $S \hookrightarrow G$ such that every prime factor of $|S|$ divides $|W|$ and $S$ has the following property.

(i) If Char $(k)$ does not divide $|W|$, then the map $H^{1}(K, S) \rightarrow H^{1}(K, G)$ is surjective for every field $K / k$; see [CGR].

(ii) If $\operatorname{char}(k)$ divides $|W|$, the above map is surjective for every perfect field $K / k$; see [CGR2, Corollary 1.4].

We fix a finite subgroup $S$ with these properties for the rest of the proof.

Let $\rho:\left(\widehat{\mathbb{Z}}^{\prime}\right)^{n} \rightarrow \operatorname{Gal}\left(F_{n}\right)$ be a splitting of the exact sequence (5.2) in Lemma 5.1. Denote the extension of $F_{n}$ associated by the Galois correspondence to the image of $\rho$ by $E_{n} / F_{n}$ and its perfect closure by $E_{n}^{\text {perf }} / F_{n}$. Note that if $\operatorname{char}(k)=0$, then $\rho$ is an isomorphism and $E_{n}^{\text {perf }}=E_{n}=F_{n}$. If $\operatorname{char}(k)=p$, then the degree of any finite subextension of $E_{n} / F_{n}$ is a power of $p$ and $\operatorname{Gal}\left(E_{n}\right)=\left(\widehat{\mathbb{Z}}^{\prime}\right)^{n}$. The same is true for the perfect closure $E_{n}^{\text {perf }} / F_{n}$.

Since Galois cohomology commutes with direct limits of fields, in order to establish parts (a) and (b) of the proposition, it suffices to show that $\gamma_{E_{n}^{\text {perf }}}$ admits reduction of structure to some abelian subgroup $A \subset S$ of rank $\leq \operatorname{trdeg}_{k}(K)$ (where $|A|$ is prime to $p$, if $p=\operatorname{char}(k)>0$ ). After replacing $K$ by its algebraic closure in $E_{n}^{\text {perf }}$, we may assume that $K$ is algebraically closed in $E_{n}^{\text {perf }}$. In particular, $K$ is perfect.

By our assumption $\gamma$ descends to some $\gamma_{K} \in H^{1}(K, G)$. On the other hand, by (ii) $\gamma_{K}$ is the image of some $\delta_{K} \in H^{1}(K, S)$. The class $\delta_{K}$ is represented by a continuous homomorphism $\psi: \operatorname{Gal}(K) \rightarrow S$. Clearly $\delta_{K}$ (and hence, $\gamma_{K}$ and $\beta_{E_{n}^{\text {perf }}}$ ) admit reduction of structure to the subgroup $A=\operatorname{Im}(\psi)$ of $S$. It remains to show that $A$ is an abelian group of rank $\leq d$ whose order is prime to $p$.

By Lemma 6.2 we can identify $\operatorname{Gal}(K)$ with a quotient of $\operatorname{Gal}\left(E_{n}^{\text {perf }}\right)=\left(\widehat{\mathbb{Z}}^{\prime}\right)^{n}$. In particular, $\operatorname{Gal}(K)$ is finitely generated, abelian, and the order of every finite quotient of $\operatorname{Gal}(K)$ is prime to $p$. Moreover, by Tsen's theorem, $\operatorname{cd}(\operatorname{Gal}(K)) \leq d$; $\operatorname{cf}$. [Se, II.4.2]. Thus Lemma 6.3 enables us to conclude that $\mathrm{Gal}(K)$ is a direct summand of $\left(\widehat{\mathbb{Z}}^{\prime}\right)^{d}$. Hence, the finite quotient $A$ of $\operatorname{Gal}(K)$ is an abelian group of rank $\leq d$ whose order is prime to $p$.

6.4 Remark. A minor modification of the above argument (in particular, using (i) instead of (ii)) shows that the assertion of Proposition 6.1 (a) holds whenever Char $(k)$ does not divide the order of the Weyl group $W$ of $G$. In other words, in this case we can take $F^{\prime}$ to be $F_{n}$ in part (b). Since we will not use this result in the sequel, we leave the details of its proof as an exercise for an interested reader. 


\section{Fixed points in homogeneous spaces}

Let $k$ is an algebraically closed field of characteristic $p \geq 0, t_{1}, \ldots t_{n}$ are independent variables over $k$, and $H=(\mathbb{Z} / m \mathbb{Z})^{n}$. If $p>0$, we will assume that $m$ is prime to $p$. We will continue to denote the iterated power series field $k\left(\left(t_{1}\right)\right)\left(\left(t_{2}\right)\right) \ldots\left(\left(t_{n}\right)\right)$ by $F_{n}$.

The purpose of this section is to establish the following fixed point result which will be used in the proof of Theorem 1.2. For notational convenience, we will consider an arbitrary (not necessarily injective) morphism

$$
\phi: H=(\mathbb{Z} / m \mathbb{Z})^{n} \rightarrow G
$$

of algebraic groups. This is slightly more general than considering a finite abelian subgroup of $G$. We will assume that $G, H$ and $\phi$ are fixed throughout this section.

\subsection{Proposition. Assume that}

(1) $F^{\prime} / F_{n}$ is a finite field extension of degree prime to $|H|$,

(2) $\beta \in H^{1}\left(F_{n}, H\right)$ is represented by an $H$-Galois field extension $E / F$, and

(3) $\phi_{*}(\beta)_{F^{\prime}} \in H^{1}\left(F^{\prime}, G\right)$ admits reduction of structure to a finite subgroup $S$ of $G$.

Then $\phi(H)$ has a fixed $k$-point in any $G$-equivariant compactification $Y$ of $G / S$.

Here by a $G$-equivariant compactification of $G / S$ we mean a complete (but not necessarily smooth) $G$-variety, which contains $G / S$ as a dense open $G$-subvariety.

Proof. By Corollary 5.4, $F^{\prime}$ is $k$-isomorphic to $F_{n}$. Thus, after replacing $F_{n}$ by $F^{\prime}$ and $\beta$ by $\beta_{F^{\prime}}$, we may assume that $F^{\prime}=F_{n}$. (Note that $\beta_{F^{\prime}} \in H^{1}\left(F^{\prime}, H\right)$ is represented by the $H$-Galois algebra $E_{F^{\prime}} / F^{\prime}$, where $E_{F^{\prime}}=E \otimes_{F} F^{\prime}$. Since $E$ is a field and $\left[F_{n}^{\prime}: F_{n}\right]$ is prime to $|H|=[E: F], E_{F^{\prime}}$ is again a field.)

By Lemma 5.1, we may assume that $E=k\left(\left(s_{1}\right)\right) \ldots\left(\left(s_{n}\right)\right)$, where $s_{i}^{m}=t_{i}$ and there exists a minimal set of generators $\tau_{1}, \ldots, \tau_{n}$ of $H$ such that $H$ acts on $k\left(\left(s_{1}\right)\right) \ldots\left(\left(s_{n}\right)\right)$ by

$$
\tau_{i}\left(s_{j}\right)= \begin{cases}\zeta s_{j} & \text { if } i=j, \\ s_{j} & \text { if } i \neq j,\end{cases}
$$

where $\zeta$ is a primitive $m$ th root of unity (independent of $i$ and $j$ ). In the sequel we will denote $E$ by $F_{n, m}$; note that we previously encountered this field in the proof of Lemma 5.1.

Set $\gamma=\phi_{*}(\beta) \in H^{1}\left(F_{n}, G\right)$ and consider the twisted $F_{n}$-variety $\gamma Y$ which is a compactification of the twisted variety $\gamma(G / S)$. By our assumption $\gamma(G / S)$ has a $F_{n}$-point, so a fortiori

$$
{ }_{\gamma} Y\left(F_{n, m}\right)=\left\{y \in Y\left(F_{n, m}\right) \mid \gamma(\sigma) .^{\sigma} y=y \text { for all } \sigma \in H\right\} \neq \emptyset .
$$


Since $Y$ is complete, this implies $\gamma Y\left(F_{n-1, m}\left[\left[\sqrt[m]{t_{n}}\right]\right]\right) \neq \emptyset$. Specializing $t_{n}$ to 0 , we see that

$$
\left\{y \in Y\left(F_{n-1, m}\right) \mid \gamma(\sigma) .^{\sigma} y=y \text { for all } \sigma \in H\right\} \neq \emptyset,
$$

where the Galois action of $H$ on $Y\left(F_{n-1, m}\right)$ is induced by the canonical projection $H \rightarrow \operatorname{Gal}\left(F_{n, m} / F_{n}\right) \rightarrow \operatorname{Gal}\left(F_{n-1, m} / F_{n-1}\right)$. Repeating this process, we finally obtain

$$
\left\{y \in Y(k) \mid \gamma(\sigma) .^{\sigma} y=y \text { for all } \sigma \in H\right\} \neq \varnothing .
$$

Since $k$ is algebraically closed, we conclude that $\phi(H)$ fixes some $k$-point of $Y$.

7.4 Corollary. Let $k$ be an algebraically closed field and $G / k$ be a connected reductive group. Suppose there exists a class $\beta \in H^{1}\left(F_{n}, H\right)$ such that $\beta$ is represented by an $H$-Galois field extension of $F_{n}$. If $\phi_{*}(\beta) \in H^{1}\left(F_{n}, G\right)$ descends to a $k$-subfield $K \subset F_{n}$, then

$$
\operatorname{trdeg}_{k}(K) \geq \operatorname{rank} \phi(H)-\operatorname{rank} C_{G}(\phi(H))^{0} .
$$

Here $H$ and $\phi$ are as in (7.1).

Proof. Let $\operatorname{trdeg}_{k}(K)=d$. By Proposition 6.1 there exists a finite extension $F^{\prime} / F_{n}$ and a finite abelian subgroup $A \subset G$ of rank $\leq d$ such that

- $|A|$ is prime to char $(k)$,

- $F^{\prime}=F_{n}$ if $\operatorname{char}(k)=0$, and $\left[F^{\prime}: F_{n}\right]$ is a power of $p$ if $\operatorname{char}(k)=p$, and

- $\phi_{*}(\beta)_{F^{\prime}}$ admits reduction of structure to $A$.

Let $\bar{G}$ be a regular compactification of $G$. By Proposition 7.2, $Y=\bar{G} / A$ has a $\phi(H)$-fixed point. Now Proposition 3.2, with $\Gamma_{1}=\phi(H)$ and $\Gamma_{2}=A$, tells us that

$$
\operatorname{rank} \phi(H)-\operatorname{rank} A \leq \operatorname{rank} C_{G}(\phi(H))^{0} .
$$

Consequently,

$$
d \geq \operatorname{rank} A \geq \operatorname{rank} \phi(H)-\operatorname{rank} C_{G}(\phi(H))^{0},
$$

as claimed.

\section{Proof of Theorem 1.2}

In the statement of Theorem 1.2, we assume that $H$ is a subgroup of $G$, where as in the previous section we worked with a homomorphism $\phi: H \rightarrow G$ instead. For notational consistency, we will restate Theorem 1.2 in the following (clearly equivalent) form. 
8.1 Theorem. Let $G$ be a connected reductive linear algebraic group defined over an algebraically closed base field $k, H \simeq(\mathbb{Z} / m \mathbb{Z})^{n}$ and $\phi: H \rightarrow G$ be a (not necessarily injective) homomorphism of algebraic groups. Assume char( $k)$ does not divide $m$. Then

(a) $\operatorname{ed}(G) \geq \operatorname{rank} \phi(H)-\operatorname{rank} C_{G}(\phi(H))^{0}$.

(b) Moreover, if $H$ is an l-group (i.e., $m$ is a power of a prime integer l) then $\operatorname{ed}(G ; l) \geq \operatorname{rank} \phi(H)-\operatorname{rank} C_{G}(\phi(H))^{0}$.

Let $t_{1}, \ldots, t_{n}$ be independent variables over $k, K_{n}=k\left(t_{1}, \ldots, t_{n}\right)$ and $K_{n, m}=$ $k\left(s_{1}, \ldots, s_{n}\right)$, where $s_{i}^{m}=t_{i}$. The $H$-Galois field extension $K_{n, m} / K_{n}$ gives rise to a class $\alpha \in H^{1}\left(K_{n}, H\right)$. We will be interested in the class $\phi_{*}(\alpha) \in H^{1}\left(K_{n}, G\right)$, which we will sometimes refer to as a loop torsor. (Such torsors naturally come up in connection with loop algebras; see [GP].) We are now ready to proceed with the proof of Theorem 8.1.

(a) By the definition of $\operatorname{ed}(G)$, it suffices to show that

$$
\operatorname{ed} \phi_{*}(\alpha) \geq \operatorname{rank} \phi(H)-\operatorname{rank} C_{G}(\phi(H))^{0} .
$$

Let $d=\operatorname{ed}\left(\phi_{*}(\alpha)\right)$. Let $\beta=\alpha_{F_{n}} \in H^{1}\left(F_{n}, H\right)$. Then $\beta$ is represented by the field extension $F_{n, m} / F_{n}$. Moreover, $\phi_{*}(\beta)$ descends to $\phi^{*}(\alpha) \in H^{1}\left(K_{n}, G\right)$, which, by our assumption, further descends to a $k$-subfield of $K_{n}$ of transcendence degree $d$. Corollary 7.4 now tells us that

$$
d \geq \operatorname{rank} \phi(H)-\operatorname{rank} C_{G}(\phi(H))^{0} .
$$

This completes the proof of Theorem 8.1 (a).

(b) Once again, we will denote the class of the $H$-Galois field extension $K_{n, m} / K_{n}$ in $H^{1}\left(K_{n}, H\right)$ by $\alpha$ and consider the loop torsor $\phi_{*}(\alpha) \in H^{1}\left(K_{n}, G\right)$. By the definition of $\operatorname{ed}(G ; l)$ it suffices to show that

$$
\operatorname{ed}\left(\phi_{*}(\alpha) ; l\right) \geq \operatorname{rank} H-\operatorname{rank} C_{G}(H)^{0} .
$$

Equivalently, we want to show that

$$
\operatorname{ed}\left(\phi_{*}(\alpha)_{E}\right) \geq \operatorname{rank} H-\operatorname{rank} C_{G}(H)^{0}
$$

for every finite extension $E / K_{n}$ of degree prime to $l$. Suppose $E / K_{n}$ is such an extension and $\operatorname{ed}\left(\phi_{*}(\alpha)_{E}\right)=d$.

By [KM, Lemma 3.1] there exists a finite field extension $F^{\prime} / F_{n}$ of degree prime 
to $l$ and a $K_{n}$-embedding $E \hookrightarrow F^{\prime}$ such that the diagram

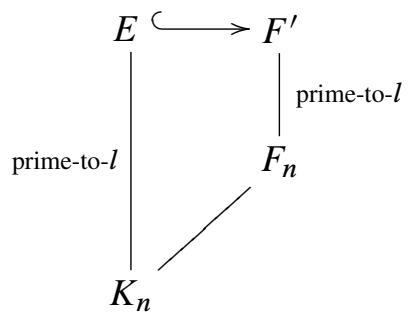

commutes. We want to conclude that $d \geq \operatorname{rank} H-\operatorname{rank} C_{G}(H)^{0}$ by applying Corollary 7.4 to $\beta=\alpha_{F^{\prime}} \in H^{1}\left(F^{\prime}, H\right)$. Since $F^{\prime}$ is $k$-isomorphic to $F_{n}$ (see Corollary 5.4), Corollary 7.4 can be applied to this $\beta$, as long as we can show that

(i) $\phi_{*}(\beta)$ descends to a $k$-subfield of $F^{\prime}$, of transcendence degree $d$ over $k$, and

(ii) $\beta$ is represented by an $H$-Galois field extension of $F^{\prime}$.

(i) is clear since $\phi^{*}(\beta) \in H^{1}\left(F^{\prime}, G\right)$ descends to $\phi^{*}(\alpha) \in H^{1}(E, G)$, which, by our assumption, descends to a $k$-subfield $E_{0} \subset E$ with $\operatorname{trdeg}_{k}\left(E_{0}\right)=d$. To prove (ii), note that $\alpha_{F_{n}}$ is represented by the field extension $F_{n, m} / F_{n}$. Thus $\beta=\alpha_{F^{\prime}}$ is represented by the $H$-Galois algebra $F_{n, m} \otimes_{F_{n}} F^{\prime}$ over $F^{\prime}$. Since $\left[F^{\prime}: F_{n}\right]$ is a finite and prime to $l, F_{n, m} \otimes F_{n} F^{\prime}$ is a field. This concludes the proof of (ii) and thus of Theorem 8.1 (b).

\section{Examples}

This section contains five examples illustrating Theorem 1.2(b).

9.1 Example. If $\operatorname{char}(k) \neq 2$, then $\operatorname{ed}\left(\mathrm{GO}_{n} ; 2\right) \geq n-1$.

Proof. Let $H \simeq(\mathbb{Z} / 2 \mathbb{Z})^{n}$ be the subgroup of diagonal matrices in $\mathrm{O}_{n}$. Viewing $H$ as a subgroup of $\mathrm{GO}_{n}$, we easily see that $C_{\mathrm{GO}_{n}}^{0}(H)=$ the center of $\mathrm{GO}_{n}$, has rank 1 . Applying Theorem 1.2 (b) to this subgroup we obtain the desired bound.

9.2 Example. If $p$ is a prime and $\operatorname{char}(k) \neq p$, then

(a) $\operatorname{ed}\left(\mathrm{SL}_{p^{r}} / \mu_{p^{s}} ; p\right) \geq \begin{cases}2 s+1 & \text { if } s<r, \\ 2 s & \text { if } s=r ;\end{cases}$
(b) $\operatorname{ed}\left(\mathrm{GL}_{p^{r}} / \mu_{p^{s}} ; p\right) \geq \begin{cases}2 s, & \text { if } s<r, \\ 2 s-1 & \text { if } s=r .\end{cases}$ 
Proof. (a) The group $\mathrm{SL}_{p^{r}} / \mu_{s}$ has a self-centralizing subgroup

$$
H \simeq(\mathbb{Z} / p \mathbb{Z})^{r} \times \mathbb{Z} / p^{r-s} \mathbb{Z}
$$

see $\left[\mathrm{RY}_{1}\right.$, Lemma 8.12]. Now apply Theorem 1.2 (b) to this group.

(b) We now view $H$ as a subgroup of $\mathrm{GL}_{p^{r}} / \mu_{p^{s}}$. The centralizer $C_{\mathrm{GL}_{p^{r}} / \mu_{s}}^{0}(H)$ is the center of $\mathrm{GL}_{p^{r}} / \mu_{s}$; it is isomorphic to a 1-dimensional torus. Part (b) now follows from Theorem 1.2(b).

The non-vanishing of the Rost invariant $H^{1}(*, G) \rightarrow H^{3}\left(*, \mu_{p}\right)$ for a group $G$ and a prime $p$ implies that $\operatorname{ed}(G ; p) \geq 3$; cf. [Re, Theorem 12.14]. In particular, one can show that ed $\left(F_{4} ; 3\right)$, ed $\left(E_{6} ; 2\right)$ and $\operatorname{ed}\left(E_{7} ; 3\right) \geq 3$ in this way. In Examples 9.3-9.6 below we will deduce these inequalities directly from Theorem $1.2(\mathrm{~b})$ and show that equality holds in each case.

9.3 Example. If $\operatorname{char}(k) \neq 3$, then $\operatorname{ed}\left(F_{4} ; 3\right)=3$.

Proof. $F_{4}$ has a self-centralizing subgroup isomorphic to $\left(\mu_{3}\right)^{3}$; see [Gr, Theorem 7.4]. Theorem 1.2 now tells us that $\operatorname{ed}\left(F_{4} ; 3\right) \geq 3$. To prove the opposite inequality, recall that $H^{1}\left(K, F_{4}\right)$ classifies the exceptional 27-dimensional Jordan algebras $J / K$. After a quadratic extension $K^{\prime} / K, J \otimes_{K} K^{\prime}$ is given by the first Tits construction. Without loss of generality, we may assume that $J=(A, v)$ where $A$ is a central simple $K$-algebra of degree 3 and $v$ is a scalar in $K$. Since $A$ is a symbol algebra $(a, b)_{3}$, we see that $J \otimes_{K} K^{\prime}$ descends to the subfield $k(a, b, v)$ of $K$ of transcendence degree $\leq 3$. We conclude that $\operatorname{ed}(J ; 3) \leq 3$ and thus $\operatorname{ed}\left(F_{4} ; 3\right) \leq 3$, as claimed.

9.4 Example. If $\operatorname{char}(k) \neq 2$, then $\operatorname{ed}\left(E_{6} ; 2\right)=3$.

Here $E_{6}$ denotes the simply connected simple group of type $E_{6}$. By abuse of notation we will also write $E_{6}$ for the Dynkin diagram of $E_{6}$.

Proof. By [Gr, Table II], $E_{6}$ has a unique (up to conjugation) non-toral subgroup $H$ isomorphic to $(\mathbb{Z} / 2 \mathbb{Z})^{5}$. To compute the rank of its centralizer, we make use of its Witt-Tits index $I(H) \subset E_{6}$ which describes the type of a minimal parabolic subgroup containing $H$; see [GP, Section 3]. The Dynkin diagram for $E_{6}$ is as follows.

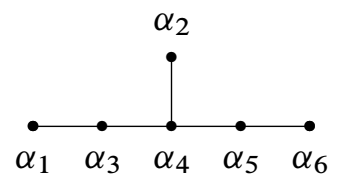


Set $I=\left\{\alpha_{2}, \alpha_{3}, \alpha_{4}, \alpha_{5}\right\}$. Let $P_{I}$ be a standard parabolic subgroup and $L_{I}=Z_{G}\left(T_{I}\right)$ its standard Levi subgroup. Then $D L_{I}=\operatorname{Spin}_{8}$. Since $\operatorname{Spin}_{8}$ has a maximal nontoral 2-elementary abelian subgroup of rank 5 (see [Gr, Table I]), we may assume that $H \subset \operatorname{Spin}_{8}$. Moreover, $C_{\mathrm{Spin}_{8}}(H)$ is finite, so $H$ is irreducible in $L_{I}$. It follows that $P_{I}$ is a minimal parabolic subgroup of $E_{6}$ containing $H$; the Witt-Tits index of $H$ is then $I$. By [GP, Proposition 3.11], we have

$$
\operatorname{rank} C_{E_{6}}(H)=\left|E_{6} \backslash I\right|=2 .
$$

Theorem 1.2 (b) now tells us that ed $\left(E_{6} ; 2\right) \geq 5-2=3$. (In [GP] the base field is assumed to be of characteristic 0. However, in our situation, [GP, Proposition 3.11] applies as long as $\operatorname{char}(k) \neq 2$.)

To prove the opposite inequality, suppose $\alpha \in H^{1}\left(K, E_{6}\right)$, where $K$ is a field containing $k$. Let $L \subset E_{6}$ be the Levi subgroup of the parabolic $E_{6} \backslash\left\{\alpha_{6}\right\}$. We observe that the finite groups $N_{L}(T) / T$ and $N_{E_{6}}(T) / T$ have isomorphic 2-Sylow subgroups of order $2^{7}$. By [Gi, Lemme 3.a], it follows that there exists a finite odd degree extension $K^{\prime} / K$ such that $\alpha_{K^{\prime}}$ belongs to the image of $H^{1}\left(K^{\prime}, L\right) \rightarrow H^{1}\left(K^{\prime}, E_{6}\right)$. Hence the class $\alpha_{K^{\prime}}$ is isotropic with respect to the root $\alpha_{6}$. By the list of Witt-Tits indices in [T], the class $\alpha_{K^{\prime}}$ is isotropic with respect to $I$. So $\alpha_{K^{\prime}}$ belongs to the image of $H^{1}\left(K^{\prime}, L_{I}\right) \cong H^{1}\left(K^{\prime}, P_{I}\right) \rightarrow H^{1}\left(K^{\prime}, E_{6}\right)$.

It thus remains to show that

$$
\operatorname{ed}\left(L_{I} ; 2\right) \leq 3
$$

To prove this inequality, we need an explicit description of the group $L_{I}$. Recall that there is a natural inclusion $\mu_{2} \times \mu_{2}=C\left(D L_{I}\right) \subset T_{I}=\mathbb{G}_{m} \times \mathbb{G}_{m}$; see [CP, Proof of Proposition 14.a]. Hence we have the following commutative exact diagram:

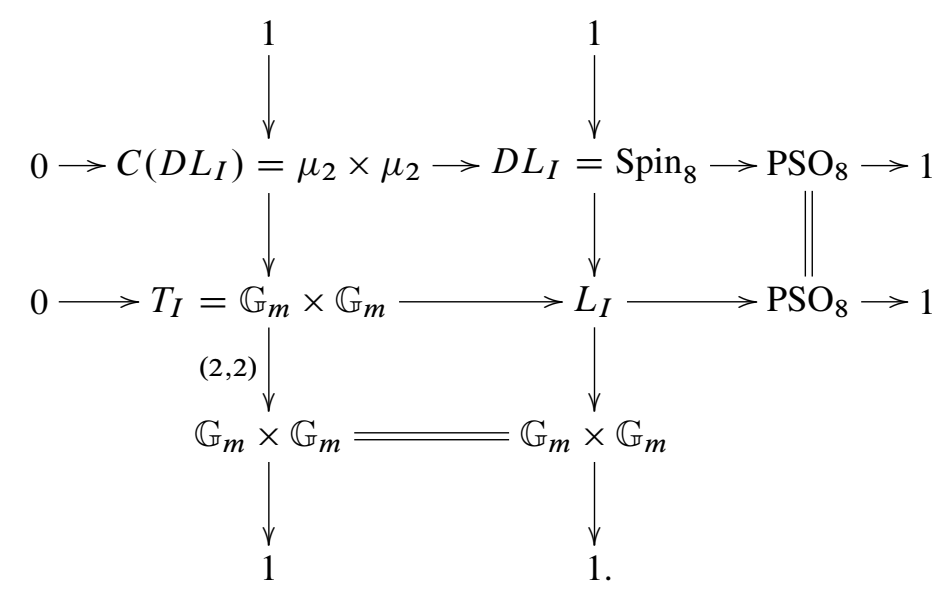

Taking Galois cohomology of the right square over a field $F / k$, we obtain the fol- 
lowing commutative exact diagram of pointed sets:

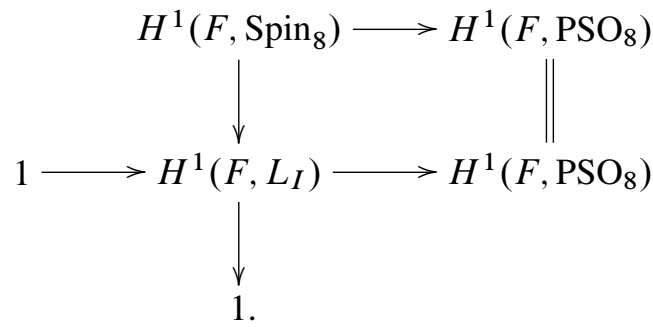

By the usual twisting argument, $H^{1}\left(F, L_{I}\right)$ injects into $H^{1}\left(F, \mathrm{PSO}_{8}\right)$, therefore $H^{1}\left(F, L_{I}\right)=\operatorname{Im}\left(H^{1}\left(F, \operatorname{Spin}_{8}\right) \rightarrow H^{1}\left(F, \mathrm{PSO}_{8}\right)\right)$. It is well known that the image of $H^{1}\left(F, \mathrm{Spin}_{8}\right)$ in $H^{1}\left(F, \mathrm{PSO}_{8}\right)$ classifies the similarity classes of 8-dimensional quadratic $F$-forms in $I^{3}(F)$; cf., e.g., [KMRT, pp. 409 and 437]. By the ArasonPfister Theorem, every 8-dimensional quadratic form $q \in I^{3}(F)$ is similar to a 3-fold Pfister form $\langle\langle a, b, c\rangle\rangle$. Thus the similarity class of $q$ is defined over $k(a, b, c)$. This shows that $\operatorname{ed}\left(L_{I} ; 2\right) \leq 3$, as claimed.

9.5 Remark. One can show that for every $\alpha \in H^{1}\left(K, E_{6}\right)$ there is an odd degree field extension $L / K$ such that $\alpha_{L}$ lies in the image of the natural map $H^{1}\left(L, G_{2}\right) \rightarrow$ $H^{1}\left(L, E_{6}\right)$; see [GMS, Exercise 22.9]. Since ed $\left(G_{2}\right)=3$, this leads to an alternative proof of the inequality ed $\left(E_{6} ; 2\right) \leq 3$.

9.6 Example. If $\operatorname{char}(k) \neq 3$, then $\operatorname{ed}\left(E_{7} ; 3\right)=3$.

Here $E_{7}$ denotes the simply connected simple group of type $E_{7}$. By abuse of notation we will sometimes also write $E_{7}$ for the Dynkin diagram of $E_{7}$.

Proof. By [Gr, Table III], $E_{7}$ has a unique (up to conjugation) non-toral subgroup $H$ isomorphic to $(\mathbb{Z} / 3 \mathbb{Z})^{5}$. To compute the rank of its centralizer, we make use of its Witt-Tits index $I(H) \subset E_{7}$ [GP, Section 3], where $E_{7}$ is the following Dynkin diagram.

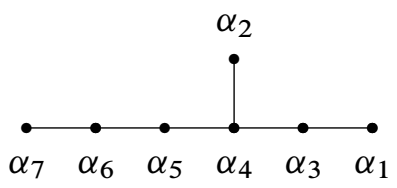

Set $I=E_{7} \backslash\left\{\alpha_{7}\right\}$ and let $P_{I}$ be the standard parabolic subgroup. Denote by $L_{I}=Z_{G}\left(T_{I}\right)$ its standard Levi subgroup. Then $D L_{I}=E_{6}$, where $E_{6}$ denotes a simply connected group of type $E_{6}$. Since $E_{6}$ has a maximal non-toral 3-elementary abelian subgroup of rank 4 (see [Gr, Table III]), we may assume that $H \subset E_{6}$. 
Moreover, $C_{E_{6}}(H)$ is finite, so $H$ is irreducible in $L_{I}$. It follows that $P_{I}$ is a minimal parabolic subgroup of $E_{7}$ containing $H$ and thus the Witt-Tits index of $H$ is $I$. By [GP, Proposition 3.19], the group $C_{E_{7}}(H)$ is of rank 1. Theorem 1.2 (b) now tells us that ed $\left(E_{7} ; 3\right) \geq 4-1=3$.

To prove the opposite inequality, consider $\alpha \in H^{1}\left(K, E_{7}\right)$. By [Ga, Example 3.5], the natural map

$$
H^{1}\left(K, E_{6} \rtimes \mu_{4}\right) \rightarrow H^{1}\left(K, E_{7}\right)
$$

is surjective. Here, once again, $E_{6}$ stands for the simply connected group of type $E_{6}$. It follows that there exists a quartic extension $K^{\prime} / K$ such that $\alpha_{K^{\prime}}$ admits reduction of structure to $E_{6}$ (i.e., lies in the image of the map $H^{1}\left(K^{\prime}, E_{6}\right) \rightarrow H^{1}\left(K^{\prime}, E_{7}\right)$ ). Thus we may assume without loss of generality that $\alpha$ comes from $E_{6}$. Now recall that the natural map $H^{1}\left(K, F_{4} \rtimes \mu_{3}\right) \rightarrow H^{1}\left(K, E_{6}\right)$ is surjective (see [Ga, Example 3.5]); here $\mu_{3}=C\left(E_{6}\right)$. Thus there exists a $\beta \in H^{1}\left(K, F_{4} \rtimes \mu_{3}\right)$ mapping to $\alpha$ by the composite map

$$
H^{1}\left(K, F_{4} \rtimes \mu_{3}\right) \rightarrow H^{1}\left(K, E_{6}\right) \rightarrow H^{1}\left(K, E_{7}\right) .
$$

We claim that $\alpha \in H^{1}\left(K, E_{7}\right)$ admits further reduction of structure to $F_{4}$ (i.e., $\alpha$ depends only of the $F_{4}$-component of $\beta$ ). If this claim is established the desired inequality ed $(\alpha ; 3) \leq 3$ will immediately follow from Example 9.3.

To prove the claim we again view $E_{6}$ inside $E_{7}$ as $E_{6}=D L_{I}$. We have $L_{I}=$ $C_{E_{7}}\left(T_{I}\right)=D L_{I} \cdot T_{I}$, where $T_{I}=\mathbb{G}_{m}$ is the standard torus associated to $I$ and $C\left(L_{I}\right)=T_{I} . C\left(E_{7}\right)$. Since $C\left(E_{7}\right)=\mu_{2}$, it follows that $\mu_{3}=C\left(E_{6}\right) \subset T_{I} \subset L_{I}$. We consider the commutative diagram of pairings

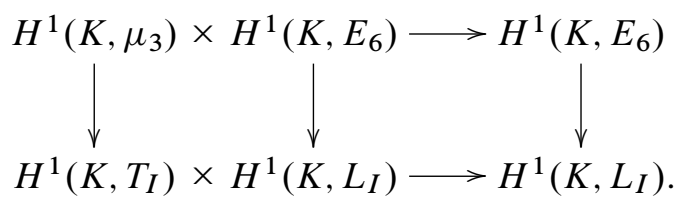

From the vanishing of $H^{1}\left(K, T_{I}\right)$, it follows that the map $H^{1}\left(K, E_{6}\right) \rightarrow H^{1}\left(K, L_{I}\right)$ is $H^{1}\left(K, \mu_{3}\right)$-invariant. A fortiori, the image of the map

$$
H^{1}\left(K, F_{4}\right) \rightarrow H^{1}\left(K, E_{6}\right) \rightarrow H^{1}\left(K, L_{I}\right)
$$

is $H^{1}\left(K, \mu_{3}\right)$-invariant. We conclude that the image of $\beta \in H^{1}\left(F_{4} \times \mu_{3}\right)$ in $H^{1}\left(K, L_{I}\right)$ depends only of its $F_{4}$ component, as claimed.

9.7 Remark. One can show that if $G \rightarrow G^{\prime}$ is a central isogeny of degree $d$, then $\operatorname{ed}(G ; p)=\operatorname{ed}\left(G^{\prime} ; p\right)$ for any prime $p$ not dividing $d$. In particular, the equalities $\operatorname{ed}\left(E_{6} ; 2\right)=3$ and ed $\left(E_{7} ; 3\right)=3$ are valid for adjoint $E_{6}$ and $E_{7}$ as well. 
Acknowledgements. We would like to thank J.-P. Serre for sharing his conjecture with us and for numerous insights related to the subject matter of this paper. We are also grateful to M. Brion, P. Brosnan, V. Chernousov and O. Gabber for helpful comments.

The second author would like to thank Laboratoire de mathématiques at the Université Paris-Sud for their hospitality during the week of June 19-25, 2006, when the work on this collaborative project began.

\section{References}

[BF] G. Berhuy, G. Favi, Essential dimension: a functorial point of view (after A. Merkurjev). Doc. Math. 8 (2003), 279-330. Zbl 1101.14324 MR 2029168

[BDP] E. Bifet, C. De Concini and C. Procesi, Cohomology of regular embeddings. Adv. Math. 82 (1) (1990), 1-34. Zbl 10743.14018 MR 1057441

[Br] M. Brion, The behaviour at infinity of the Bruhat decomposition. Comment. Math. Helv. 73 (1) (1998), 137-174. Zbl 0935.14029 MR 1610599

[BK] M. Brion, S. Kumar, Frobenius splitting methods in geometry and representation theory. Progr. Math. 231, Birkhäuser, Boston, MA, 2005. Zbl 1072.14066 MR 2107324

[CGR] V. Chernousov, Ph. Gille, Z. Reichstein, Resolving G-torsors by abelian base extensions. J. Algebra 296 (2006), 561-581. Zbl 05024296 MR 2201056

[CGR2] V. Chernousov, Ph. Gille, Z. Reichstein, Reduction of structure for torsors over semilocal rings. Manuscripta Math. 126 (2008), 465-480. Zbl 05346202 MR 2425436

[CP] V. Chernousov, V. P. Platonov, The rationality problem for semisimple group varieties. J. Reine Angew. Math. 504 (1998), 1-28. Zbl 0904.20036 MR 1656830

[CS] V. Chernousov, J.-P. Serre, Lower bounds for essential dimensions via orthogonal representations. J. Algebra 305 (2) (2006), 1055-1070. Zbl 05078318 MR 2266867

[Co] I. S. Cohen, On the structure and ideal theory of complete local rings. Trans. Amer. Math. Soc. 59 (1946), 54-106. Zbl 0060.07001 MR 0016094

[DP] C. De Concini and C. Procesi, Complete symmetric varieties. In Invariant theory (Montecatini, 1982), Lecture Notes in Math. 996, Springer-Verlag, Berlin 1983, 1-44. Zbl 0581.14041 MR 0718125

[F] I. B. Fesenko, Complete discrete valuation fields. Abelian local class field theories. In Handbook of algebra, Vol. 1, North-Holland, Amsterdam 1996, 221-268. Zbl 0860.11069 MR 1421803

[Ga] S. Garibaldi, The Rost invariant has trivial kernel for quasi-split groups of low rank. Comment. Math. Helv. 76 (2001), 684-711. Zbl 1001.20042 MR 1881703

[GMS] S. Garibaldi, A. Merkurjev, J.-P. Serre, Cohomological invariants in Galois cohomology. Univ. Lecture Ser. 28, Amer. Math. Soc., Providence, RI, 2003. Zbl 01959122 MR 1999383

[Gi] P. Gille, Cohomologie galoisienne des groupes quasi-déployés sur des corps de dimension cohomologique $\leq$ 2. Compositio Math. 125 (2001), 283-325. Zbl 1017.11019 MR 1818983 
[GP] P. Gille and A. Pianzola, Galois cohomology and forms of algebras over Laurent polynomial rings. Math. Ann. 338 (2) (2007), 497-543. Zbl 1131.11070 MR 2302073

[Gr] R. L. Griess, Jr., Elementary abelian $p$-subgroups of algebraic groups. Geom. Dedicata 39 (1991), 253-305. Zbl 0733.20023 MR 1123145

[H] J. E. Humphreys, Linear algebraic groups. Grad. Texts in Math. 21, Springer-Verlag, New York 1975. Zbl 0325.20039 MR 0396773

[J] J. C. Jantzen, Nilpotent orbits in representation theory. In Lie theory, Progr. Math. 228, Birkhäuser, Boston, MA, 2004, 1-211. Zbl 02160654 MR 2042689

[KM] N. A. Karpenko, A. S. Merkurjev, Canonical p-dimension of algebraic groups. Adv. Math. 205 (2) (2006), 410--433. Zbl 1119.14041 MR 2258262

[KMRT] M.-A. Knus, A. Merkurjev, M. Rost, J.-P. Tignol, The book of involutions. Amer. Math. Soc. Colloq. Publ. 44, Amer. Math. Soc., Providence, RI, 1998. Zbl 0955.16001 MR 1632779

[MFK] D. Mumford, J. Fogarty, F. Kirwan, Geometric invariant theory. Third edition, Ergeb. Math. Grenzgeb. (2) 34, Springer-Verlag, Berlin 1994. Zbl 0797.14004 MR 1304906

[N] P. E. Newstead, Introduction to moduli problems and orbit spaces. Tata Inst. Fund. Res. Lectures on Math. and Phys. 51, Tata Institute of Fundamental Research, Bombay, Narosa Publishing House, New Delhi 1978. Zbl 0411.14003 MR 0546290

[PV] V. L. Popov and E. B. Vinberg, Invariant Theory. In Algebraic Geometry IV, Encyclopedia Math. Sci. 55, Springer-Verlag, Berlin 1994, 123-284. MR 1100485

[Po] V. L. Popov, Sections in invariant theory. In The Sophus Lie Memorial Conference (Oslo, 1992), Scand. Univ. Press, Oslo 1994, 315-361. MR 1456471

[Re] Z. Reichstein, On the notion of essential dimension for algebraic groups. Transform. Groups 5 (3) (2000), 265-304. Zbl 0981.20033 MR 1780933

$\left[\mathrm{RY}_{1}\right] \quad$ Z. Reichstein and B. Youssin, Essential dimensions of algebraic groups and a resolution theorem for $G$-varieties. With an appendix by J. Kollár and E. Szabó. Canad. J. Math. 52 (5) (2000), 1018-1056, Zbl 1044.14023 MR 1782331

$\left[\mathrm{RY}_{2}\right] \quad$ Z. Reichstein and B. Youssin, Splitting fields of G-varieties. Pacific J. Math. 200 (1) (2001), 207-249. Zbl 1054.14063 MR 1863413

[RZ] L. Ribes, P. Zalesskii, Profinite Groups. Ergeb. Math. Grenzgeb. 40, Springer-Verlag, Berlin 2000. Zbl 0949.20017 MR 1775104

[Se] J.-P. Serre, Galois Cohomology. Springer-Verlag, Berlin 1997. Zbl 0902.12004 MR 1466966

[St] E. Strickland, A vanishing theorem for group compactifications. Math. Ann. 277 (1) (1987), 165-171. Zbl 0595.14037 MR 0884653

[T] J. Tits, Classification of algebraic semisimple groups. In Algebraic groups and discontinuous subgroups (eds. A. Borel and G. Mostow), Proc. Symp. Pure Math. 9, Amer. Math. Soc., Providence, RI, 1966, 33-62. Zbl 0238.20052 MR 0224710

[W] A. Wadsworth, Valuation theory on finite dimensional division algebras. In Valuation theory and its applications, (Saskatoon, SK, 1999), Vol. I, Fields Inst. Commun. 32, Amer. Math. Soc., Providence, RI, 2002, 385-449. Zbl 1017.16011 MR 1928379 
Received March 27, 2007

Philippe Gille, UMR 8552 du CNRS, DMA, Ecole Normale Supérieure, 75005 Paris, France E-mail: Philippe.Gille@ens.fr

Zinovy Reichstein, Department of Mathematics, University of British Columbia, Vancouver, BC V6T 1Z2, Canada

E-mail: reichst@math.ubc.ca 